\title{
Tradução, adaptação e validação preliminar do Prospective and Retrospective Memory Questionnaire (PRMQ) ${ }^{1}$
}

\author{
Daniela Benites \\ William B. Gomes ${ }^{2}$
}

\begin{abstract}
Resumo
Falhas de memória são comuns no cotidiano. Contudo, elas podem indicar ocorrências de problemas cognitivos, principalmente entre idosos. O artigo traz a tradução e a adaptação do Prospective and Retrospective Memory Questionnaire (PRMQ). O instrumento, no original constituído de 16 itens, avalia auto-relatos de falhas de memória prospectiva e retrospectiva. O estudo contou com 642 participantes, com idade variando entre 16 e 81 anos, recrutados em uma universidade e em grupos comunitários. A análise fatorial exploratória dos dados do PRMQ apontou a validade de construto apenas para oito itens. Então, optou-se por reconsiderar mais dois itens que apresentaram carga fatorial aceitável e compor uma escala de 10 itens, sendo cinco para cada dimensão da memória. A correlação para validade convergente e discriminante foi realizada com o Questionário de Percepção Subjetiva de Queixas de Memória para idosos (MAC-Q), em uma amostra de 38 participantes idosos, com idade média de 69 anos. O PRMQ-10 apresentou validade e confiabilidade $(\alpha=0,80)$, com boas perspectivas para uso em pesquisa.

Palavras-chave: Falhas de memória; Prospectiva; Retrospectiva.
\end{abstract}

\section{The Prospective and Retrospective Memory Questionnaire's (PRMQ) translation, adaptation and preliminary validation}

\begin{abstract}
Memory failures are common in daily life. However, these failures could indicate cognitive problems, mainly in elders. This study presents the translation and adaptation of the Prospective and Retrospective Memory Questionnaire (PRMQ) to Portuguese. The questionnaire measures prospective and retrospective self-report memory failures through 16 items. There were 642 participants aged between 16 and 81 years recruited in a university and in community groups. The exploratory factor analysis revealed the construct validity only for eight items. So, it was reconsidered two of the original 16 items that showed acceptable factorial load and tested a questionnaire of 10 items. Convergent and concurrent validity was tested in a sample of 38 participants aged around 69 years, through the Elders' Subjective Perception of Memory Complaints Questionnaire (MAC-Q). As a result the reduced PRMQ with 10 items showed both validity and confidence $(\alpha=0,80)$. At this point the questionnaire seems promising for research using.

Keywords: Memory failures; Prospective; Retrospective.
\end{abstract}

\section{Introdução}

Em virtude do declínio cognitivo relativo à idade, as falhas de memória são mais preocupantes na terceira idade, podendo indicar processos degenerativos como declínio cognitivo leve, Alzheimer e Parkinson. Falhas de memória podem estar ligadas a situações do futuro ou do passado. Dificuldades na evocação das intenções que devem ser realizadas no futuro, dentro de um espaço de tempo curto ou longo, são denominadas de falhas de memória prospectiva (MP). Dificuldades na evocação de eventos e situações prévias são denominadas de falhas de memória retrospectiva (MR).
Apesar da simplicidade dos questionários de auto-relato de falhas de memória, estes instrumentos são reconhecidos pela praticidade na obtenção de informações pessoais por serem de fácil preenchimento e interpretação (Crawford, Smith, Maylor, Sala \& Logie, 2003). Auto-relatos são necessários em baterias de avaliação neuropsicológica, pois as queixas de memória constituem um dos elementos para o diagnóstico de problemas cognitivos. O Teste de Percepção Subjetiva de Memória (MAC-Q) (Crook, Feher \& Larrabee, 1992) é um dos poucos instrumentos desenvolvidos para esse fim. O MAC-Q quantifica principalmente queixa de memória retrospectiva, deixando em aberto uma

\footnotetext{
${ }_{1}$ Pesquisa realizada com apoio da CAPES.

2 Endereço para correspondência:

Rua General Couto de Magalhães, 1.155 apto. 601 - 90540-131 - Porto Alegre-RS - Fax 51 3343-5850

E-mail: gomesw@ufrgs.br
} 
potencial contribuição da avaliação de queixas de memória prospectiva.

Memórias prospectivas envolvem a formação e execução de uma intenção futura, enquanto memórias retrospectivas referem-se a informações de eventos passados (Crawford e cols., 2003). As memórias prospectivas são formadas por quatro fases. A primeira corresponde à codificação da intenção e assemelha-se à memória retrospectiva, sendo chamada de componente retrospectivo da intenção. A segunda fase abrange o intervalo, curto ou longo, entre a codificação e a lembrança da intenção. Essa fase é característica da memória prospectiva, distingue-a de processos como a resolução de problemas e vigilância, e representa o componente prospectivo da intenção (Brandimonte, Ferrante, Feresin \& Delbello, 2001). Na terceira fase, a intenção é evocada e executada. Na quarta fase, a intenção deve ser cancelada (Parente, Taussik, Ferreria \& Kristensen, 2005). A falta de cancelamento reativa a terceira fase, e a intenção pode ser novamente executada sem necessidade, como quando alguém volta para apagar a luz que já havia apagado. As memórias retrospectivas são formadas através do armazenamento, codificação e recuperação de eventos passados (Henry, MacLeod, Phillips \& Crawford, 2004).

Smith, Sala, Logie \& Maylor (2000) desenvolveram um modelo para estudo de falhas de memória prospectiva (MP) e memória retrospectiva (MR), viabilizado por meio de um questionário de autorelato, o Prospective and Retrospective Memory Questionnaire (PRMQ). Nesse modelo, memória retrospectiva (MR) é utilizada em situações relacionadas a fatos passados, tais como reconhecer um rosto familiar, recordar o nome de alguém ou lembrar o caminho para chegar a algum lugar. Memória prospectiva (MP), ou de intenção (Smith e cols., 2000), é aquela utilizada para realizar atividades no futuro que estão associadas a intenções prévias (Marsh, Hicks \& Watson, 2002).

O PRMQ (Smith e cols., 2000) foi elaborado para avaliar o auto-relato de falhas de memória prospectiva e retrospectiva, em populações clínicas, em portadores de déficits cognitivos e em populações saudáveis. $\mathrm{O}$ instrumento foi desenvolvido para verificar duas hipóteses: falhas de MP são mais freqüentes do que falhas de MR em pacientes com Alzheimer; falhas de MP são mais freqüentes do que falhas de MR durante o envelhecimento normal (Smith e cols., 2000).

O estudo de normatização do PRMQ (Smith e cols., 2000) demonstrou que as variáveis demográficas de idade e gênero não influenciaram os escores do questionário (Crawford e cols., 2003). A fidedignidade do PRMQ foi considerada bem aceitável para estudos grupais e individuais (Smith e cols., 2000). A estrutura do questionário compõe-se de três fatores principais: memória geral, memória prospectiva e memória retrospectiva. Todos os itens classificam um fator substancial comum de memória, a memória geral, e os itens de MP e de MR indicam os fatores de memórias específicos de cada escala. Assim, o PRMQ refletiu um modelo tripartido, no qual a memória geral, a MP e a MR são os fatores latentes, e os itens do questionário são as variáveis manifestas.

O PRMQ (Smith e cols., 2000) é uma medida de auto-avaliação, e não uma medida direta de memória. Deve ser utilizado na quantificação de queixas, na identificação de questões relevantes para uma entrevista clínica, na formação de hipóteses clínicas (Smith e cols., 2000) e na investigação da associação de diferentes variáveis com o relato de falha de memória prospectiva ou retrospectiva (Crawford e cols., 2003).

Dos 16 itens do questionário, oito avaliam o autorelato de falhas de memória prospectiva (intenções) e oito abordam o auto-relato de falhas de memória retrospectiva (eventos passados). Cada item de MP e MR é categorizado de acordo com duas subcategorias: de tempo (curto e longo prazo) e pista (interna ou externa). Assim, as escalas de auto-relato de falhas de memória prospectiva e retrospectiva possuem o mesmo número de itens sobre memória de curto e longo prazo e memória com pista externa e pista interna. Cada um dos 16 itens apresenta três dimensões; por exemplo, o item 7 (Você esquece de comprar algo que você planejou comprar, como um cartão de aniversário, mesmo que você veja a loja?) avalia memória prospectiva, de longo prazo, com pista no ambiente (externa). O escore geral máximo no PRMQ é 80 e representa um alto índice de queixas de memória; o escore geral mínimo é 16, para poucas queixas de memória.

$\mathrm{Na}$ memória prospectiva com pista externa, a lembrança da intenção está ligada a um indicador no ambiente, como um lembrete ou um sinal que representa o conteúdo da intenção. Um modelo de estudo experimental da memória prospectiva com pista externa requer que os participantes pressionem uma tecla específica do computador quando determinada palavra aparecer na tela, durante uma tarefa (qual é a tarefa) de memorização de palavras (Cherry e cols., 2001). Em contraste, os estudos com pista interna requerem a realização de uma intenção após certo período de tempo mediante um indicador autogerado. Um exemplo é o estudo que examina o comportamento de verificação das horas no relógio e mede o tempo de latência da resposta, no intervalo da tarefa prospectiva (Einstein, McDaniel, Richardson, Guynn \& Cunfer, 1995).

As pistas, externas ou internas, são responsáveis pela estimulação da lembrança de uma ação (MP) ou de

Psico-USF, v. 12, n. 1, p. 45-54, jan./jun. 2007 
um evento (MR) (Smith e cols., 2000). Foi sugerido que os processos das MP com pista externa são mais automáticos e espontâneos e que os de MP com pista interna dependem mais de auto-iniciação e processamento consciente (Einstein e cols., 1995). Na memória prospectiva, intenções de curto prazo são ações que devem ser realizadas dentro de alguns minutos ou poucas horas; intenções de longo prazo são as que devem ser realizadas em alguns dias, meses ou anos. Na memória retrospectiva, eventos de curto prazo aconteceram há alguns dias ou poucos meses, e os de longo prazo são os que aconteceram há muitos meses ou anos.

Este estudo é um trabalho de tradução e adaptação, para uso em língua portuguesa, do Prospective and Retrospective Memory Questionnaire (PRMQ) (Smith e cols., 2000), colocando à disposição da comunidade um instrumento simples e sugestivo para a avaliação de autorelatos de memória. $\mathrm{O}$ estudo também testou a validade de construto convergente e discriminante do instrumento. A maioria dos questionários de auto-relato não distingue a memória prospectiva da retrospectiva. Por exemplo, o Everyday Memory Questionnaire (Sunderland, Harris \& Baddeley, 1988) possui 28 itens, dos quais somente três avaliam a memória prospectiva. Do mesmo modo, o MAC-Q (Crook e cols., 1992), que é utilizado em pesquisas com populações brasileiras (Argimon \& Stein, 2005), possui aparentemente um item que avalia a memória prospectiva.

\section{Método}

\section{Participantes}

Os protocolos de pesquisa foram respondidos por 895 participantes. Os dados foram coletados em uma universidade privada e em instituições voltadas a atividades com grupos de terceira idade. Foram excluídos das análises participantes: com histórico de doença psiquiátrica (177), em tratamento para hipo ou hipertireoidismo (15), com má auto-avaliação de saúde geral (14) e com nível de escolaridade abaixo de quatro anos (12). Ainda, 35 protocolos não puderam ser analisados em razão do mau preenchimento, caracterizado pela omissão de respostas. Os critérios de exclusão foram utilizados para fins de homogeneização dos dados e para evitar a interferência das condições adversas de saúde nos resultados das análises estatísticas. A amostra analisada constou de 642 participantes com idade entre 16 e 81 anos, média de 26,62 anos (Desvio Padrão: 13,89), escolaridade média de 13,82 anos $(\mathrm{DP}=3,02)$ e nível socioeconômico médio segundo dados das instituições procuradas. Dos participantes 63\% eram do sexo feminino, $77,9 \%$ solteiros, tendo $87,9 \%$ deles avaliado a própria saúde como boa. Da amostra geral, foi formada uma subamostra com 38 participantes idosos (idade entre 60 e 81 anos) com idade média de 69,03 anos $(\mathrm{DP}=5,28)$, para análises exclusivas realizadas com essa faixa etária. Entre os idosos, a média de anos de educação foi 8,16 ( $\mathrm{DP}=2,34)$, sendo $89,5 \%$ do sexo feminino e, $42,1 \%$ viúvos. Desta subamostra, $54,1 \%$ disseram ter boa saúde.

\section{Instrumento}

Foram utilizados três instrumentos: Ficha de Dados Sociodemográficos; Prospective and Retrospective Memory Questionnaire (PRMQ) e Teste de Percepção Subjetiva de Memória (MAC-Q) (Crook e cols., 1992). A Ficha de Dados Sociodemográficos foi elaborada para o levantamento das características amostrais: idade, sexo, estado civil, nível de escolaridade, estado de saúde geral, e histórico de doenças e hospitalizações. O Prospective and Retrospective Memory Questionnaire (PRMQ) de Smith e cols. (2000) contém 16 itens referentes a falhas cotidianas de memória, sendo oito de memória prospectiva e oito de memória retrospectiva. Cada item é seguido de uma escala Likert de cinco pontos: (1) nunca, (2) raramente, (3) algumas vezes, (4) freqüentemente e (5) quase sempre. O escore máximo é de 80 pontos e reflete um alto índice de auto-relato de falhas de memória. O escore mínimo é de 16 pontos e corresponde a um baixo índice de auto-relato de falhas de memória. Um exemplo de item prospectivo é "Você esquece de compromissos se não for lembrado por outra pessoa ou por um lembrete, como um calendário ou agenda?" (item 5), e um exemplo de item retrospectivo "Você falha em reconhecer um lugar que você já tinha visitado antes?" (item 2). O Teste de Percepção Subjetiva da Memória (MAC-Q) de Crook e cols. (1992) é um breve questionário para acessar declínio de memória em idosos. Consta de seis itens que refletem situações abrangentes sobre o uso de memória atual, a serem comparadas com a memória aos 40 anos de idade. De acordo com os autores, a medida passou por exames de fidedignidade e confiabilidade, apresentando uma correlação significativa $(r=41, p<0,0001)$ com vários questionários de memória já validados. Os itens são avaliados segundo uma escala Likert de cinco pontos: muito melhor agora (1), um pouco melhor agora (2), sem mudança (3), um pouco pior agora (4) ou muito pior agora (5). O número máximo de pontos obtido no MACQ é 35 e reflete a percepção de muitas queixas; o mínimo de pontos é sete. Um item típico do MAC-Q é "Lembrar o nome de pessoas que acabou de conhecer" (item 1). O instrumento, utilizado apenas em participantes com idade entre 60 e 81 anos, serviu para testar a validade convergente e discriminante do PRMQ. De acordo com os autores, 
Tabela 1 - Itens e categorias do PRMQ

\begin{tabular}{|c|c|c|c|c|}
\hline $\begin{array}{c}\text { Item } \\
\mathrm{n}^{\circ}\end{array}$ & Descrição do item & $\begin{array}{c}\text { Prospectiva } v s \\
\text { retrospectiva }\end{array}$ & $\begin{array}{c}\text { Curto } v s \\
\text { longo prazo }\end{array}$ & $\begin{array}{l}\text { Pista interna } v s \\
\text { pista externa }\end{array}$ \\
\hline 1 & $\begin{array}{l}\text { Você decide fazer alguma coisa em alguns minutos e então } \\
\text { se esquece de fazê-la? }\end{array}$ & Prospectiva & Curto prazo & Pista interna \\
\hline 2 & $\begin{array}{l}\text { Você falha em reconhecer um lugar que você tenha visitado } \\
\text { antes? }\end{array}$ & Retro & Longo prazo & Pista externa \\
\hline 3 & $\begin{array}{l}\text { Você falha em fazer alguma coisa que você deveria fazer } \\
\text { poucos minutos mais tarde mesmo que esteja lá na sua } \\
\text { frente, como tomar um remédio ou apagar o fogo da } \\
\text { chaleira? }\end{array}$ & Prospectiva & Curto prazo & Pista externa \\
\hline 4 & $\begin{array}{l}\text { uece alguma coisa que lhe foi contada alguns } \\
\text { antes? }\end{array}$ & Retr & Curtc & Pista intern \\
\hline 5 & $\begin{array}{l}\text { Você esquece de compromissos se não for lembrado por } \\
\text { outra pessoa ou por um lembrete, como um calendário ou } \\
\text { agenda? }\end{array}$ & Prospectiva & Longo prazo & Pista interna \\
\hline 6 & $\begin{array}{l}\text { Você falha em reconhecer um personagem em um programa } \\
\text { de rádio ou de TV de uma cena para outra? }\end{array}$ & ectiva & Curto prazo & Pista externa \\
\hline 7 & $\begin{array}{l}\text { Você esquece de comprar algo que você planejou comprar, } \\
\text { como um cartão de aniversário, mesmo quando você vê a } \\
\text { loja? }\end{array}$ & Pros & Lonş & Pista externa \\
\hline 8 & $\begin{array}{l}\text { Você falha ao lembrar co } \\
\text { últimos dias? }\end{array}$ & Retrospectiva & Longo prazo & Pista interna \\
\hline 9 & $\begin{array}{l}\text { mesma história para a } \\
\text { rentes? }\end{array}$ & Retrospectiva & Longo prazo & Pista externa \\
\hline 10 & $\begin{array}{l}\text { Você pretende levar algo com você, antes de deixar uma sala } \\
\text { ou sair para a rua, mas minutos depois deixa o que queria } \\
\text { levar para trás, mesmo que esteja lá na sua frente? }\end{array}$ & iva & razo & terna \\
\hline 11 & $\begin{array}{l}\text { Você esquece o lugar } \\
\text { como uma revista ou }\end{array}$ & Retrc & Curto prazo & Pista interna \\
\hline 12 & diram & Prospectiva & Longo prazo & Pista externa \\
\hline 13 & $\begin{array}{l}\text { Você olha para algo sem notar que viu a mes } \\
\text { momentos antes? }\end{array}$ & ectiva & Cruto prazo & Pista \\
\hline 14 & $\begin{array}{l}\text { Se você tentasse entrar em contato com um amigo ou } \\
\text { parente que estivesse fora, você se esqueceria de tentar } \\
\text { novamente mais tarde? }\end{array}$ & Pro & Lon & Pista interna \\
\hline 15 & Você esquece o que você viu na televisão no dia anterio & Retrospectiva & Longo prazo & Pista interna \\
\hline 16 & $\begin{array}{l}\text { Você se esquece de falar para alguém algo que você queria } \\
\text { falar alguns minutos antes? }\end{array}$ & $\mathrm{P}$ & 1020 & 10 \\
\hline
\end{tabular}

\section{Procedimentos}

A aplicação dos instrumentos foi realizada coletivamente nos grupos previamente contatados. Ao início de cada encontro, o Termo de Consentimento Livre e Esclarecido era lido e explicado, assim como os objetivos da pesquisa. Logo após, os participantes recebiam as orientações quanto ao preenchimento de cada instrumento. O PRMQ (Smith e cols., 2000) era descrito como um conjunto de questões sobre falhas de memória do cotidiano comuns a todas as pessoas e o MAC-Q
(Crook e cols., 1992) como um questionário para comparação entre a memória aos 40 anos e a memória atual. O estudo foi previamente aprovado pelo Comitê de Ética em Pesquisa da Universidade Federal do Rio Grande do Sul (UFRGS) e todos os participantes deram seu consentimento por escrito. Foram seguidas as Diretrizes e Normas Regulamentadoras de Pesquisas Envolvendo Seres Humanos, do Conselho Nacional de Saúde (CNS), conforme Resolução 196/96, e do Conselho Federal de Psicologia (CFP), conforme Resolução 16/2000. 
As variáveis sociodemográficas foram avaliadas pela freqüência, média e desvio padrão. A Análise Fatorial Exploratória foi realizada através da extração dos Componentes Principais com rotação varimax (Tabachnich \& Fidell, 2001). A confiabilidade do questionário e das escalas (memória prospectiva e retrospectiva) foi examinada mediante o coeficiente alfa de Cronbach (Anastasi \& Urbina, 2000). Testes de correlação bivariada foram utilizados na análise da validade de construto e discriminante. $\mathrm{O}$ coeficiente de Spearman foi utilizado para verificar significância dos resultados. As análises foram realizadas através dos pacotes estatísticos SPSS, versão 12 e foram consideradas estatisticamente significativas as diferenças com $p<0,05$. Em termos de delineamento, o presente estudo caracteriza-se como transversal e correlacional.

\section{Resultados}

\section{Tradução do PRMQ}

O PRMQ (Smith e cols., 2000) foi traduzido através de procedimentos de translation, back translation e discussões no grupo de pesquisa, a fim de ser realizada uma adaptação para o português sensível à cultura (Fachel \& Camey, 2000; Pasquali, 2001). As fases do processo de tradução compreenderam: (1) Tradução do instrumento pelos autores da pesquisa e tradutor especialista; (2) Exame do instrumento e da tradução por grupo de psicólogos, fase correspondente à adaptação cultural; (3) Back translation realizada por professor de inglês especialista para comparação com o instrumento original, fase na qual foram realizadas adaptações lingüísticas; (4) Análise dos instrumentos por grupo de psicólogos, fase correspondente ao teste da validade de face; (5) Aplicação em 40 alunos do curso de graduação em psicologia, para a avaliação da aceitação do instrumento.

A back translation revelou a necessidade da adaptação terminológica do item 11 ("Você confunde o lugar onde recém colocou alguma coisa, como uma revista ou óculos?”). O termo mislay foi traduzido como esquece e não como confunde, pois o item correspondia originalmente a uma variável retrospectiva e a adaptação facilitaria o entendimento dos respondentes. $\mathrm{Na}$ quarta fase, os 16 itens do PRMQ foram avaliados por dois juízes psicólogos que consideraram os itens apropriados e representativos do construto investigado. A versão em português do Brasil do PRMQ foi considerada, então, com validade de face. A avaliação da aceitação do instrumento, correspondente à quinta fase da tradução, foi realizada por uma entrevista coletiva com os alunos que responderam ao questionário, logo após aplicação. Os 40 alunos que participaram dessa fase tinham entre 17 e 32 anos de idade (média de 19,27 anos e DP de 1,08 anos) e não Psico-USF, v. 12, n. 1, p. 45-54, jan./jun. 2007 apresentavam histórico de doença psiquiátrica. Por fim, foi considerado que o instrumento apresentou boa aceitabilidade e bom tempo de administração (20 minutos).

\section{Análises psicométricas}

A validade de construto da versão em português do PRMQ foi investigada mediante análise fatorial exploratória e as validades convergente e discriminante por meio de testes de correlação bivariada (Anastasi \& Urbina, 2000). Os dados do PRMQ sofreram transformação raiz quadrada a fim de adequar as características da amostra às análises estatísticas. Para a análise fatorial, foram extraídos os componentes principais com rotação varimax nos 16 itens do PRMQ em uma amostra de 642 participantes. Os dados sofreram transformação raiz quadrada a fim de serem satisfeitas condições de normalidade da amostra. Com um corte de 0,30 para inclusão de uma variável na interpretação de um fator, foram extraídos dois fatores. Duas variáveis de um total de 16 migraram das dimensões de origem e seis variáveis ficaram divididas entre as duas dimensões.

A análise fatorial realizada nos 16 itens explicou $40,84 \%$ da variância da amostra, com índice de confiabilidade alfa de Cronbach $(\alpha)$ igual a 0,87 . O fator I, formado por seis itens, foi chamado memória prospectiva, por envolver a maioria dos itens prospectivos: $1,5,9,10$, 11 e 12 (Tabela 2). O fator II, composto por quatro itens, correspondeu à memória retrospectiva: itens 2, 4, 6 e 15. Os itens 9 e 11 não apresentaram o mesmo desempenho da escala original, migrando do fator II para o fator I. Os itens 9 e 11, originalmente considerados retrospectivos (fator II), migraram de dimensão e agruparam-se no fator prospectivo (fator I). Novas análises com a exclusão dos itens 9 e 11 melhoraram a variância acumulada (42,13\%), mas diminuíram a confiabilidade no PRMQ que, com 14 itens, foi para $\alpha=0,85$. Dos 16 itens, seis se dividiram entre os dois fatores: itens 4, 7, 8, 13, 14 e 16. Análises com a exclusão desses itens melhoraram a variância acumulada $(49,87)$, mas diminuíram a confiabilidade do instrumento $(\alpha=0,75)$. Desse modo, a análise fatorial revelou que apenas oito itens da versão em português do PRMQ apresentaram validade de construto ideal na amostra investigada: itens 1, 3, 5, 10 e 12 para a dimensão prospectiva (fator I), e os itens 2, 6 e 15 para a dimensão retrospectiva (fator II).

Diante da redução do número de itens do questionário, principalmente para o fator II, reconsiderouse a inclusão dos itens 4 e 8 , por terem apresentado as maiores tendências para o fator retrospectivo. As análises foram refeitas e o instrumento ficou com cinco itens para cada fator, facilitando a comparação quantitativa entre o auto-relato de falhas de memória prospectiva e 
retrospectiva. A análise fatorial da escala com dez itens, cinco em cada dimensão, apresentou uma variância acumulada de $47,42 \%(\alpha=0,80)$. Já que as transformações dificultam a interpretação dos resultados (CallegariJacques, 2004), repetiram-se as mesmas seqüências de análises sem a utilização desse recurso. Os resultados provenientes das análises dos dados não-transformados apresentaram as mesmas tendências e aproximadamente as mesmas significâncias das análises dos dados transformados.

Tabela 2 - Componentes extraídos das análises fatoriais

\begin{tabular}{|c|c|c|c|c|c|c|c|c|c|c|}
\hline \multirow{2}{*}{$\begin{array}{l}\text { Itens do } \\
\text { PRMQ }\end{array}$} & \multicolumn{2}{|c|}{16 itens } & \multicolumn{2}{|c|}{$\begin{array}{l}\text { Exclusão dos } \\
\text { itens } 9 \text { e } 11\end{array}$} & \multicolumn{2}{|c|}{$\begin{array}{l}\text { Exclusão dos } \\
\text { itens divididos }\end{array}$} & \multicolumn{2}{|c|}{$\begin{array}{l}\text { Inclusão dos itens } \\
\qquad 4 \text { e } 8\end{array}$} & \multicolumn{2}{|c|}{$\begin{array}{l}\text { Dados não } \\
\text { transformados* }\end{array}$} \\
\hline & Fator 1 & Fator 2 & Fator 1 & Fator 2 & Fator 1 & Fator 2 & Fator 1 & Fator 2 & Fator 1 & Fator 2 \\
\hline 1 & ,583 & & 638 & & 683 & & ,664 & & ,664 & \\
\hline 2 & & 609, & &, 565 & & 638, & & ,557 & & ,536 \\
\hline 3 & ,494 & ,349 & ,550 & & 608, & & ,597, & & 625, & \\
\hline 4 & & ,552 & ,317 & ,537 & & & ,327 & ,572 & ,325, & 579, \\
\hline 5 & ,522 & & ,577, & & 615, & & 602, & & 612, & \\
\hline 6 & & ,700 & & ,736 & & ,803 & & ,722 & & ,726 \\
\hline 7 & ,414 & ,378 & ,441 & ,361 & & & & & & \\
\hline 8 & ,386 & ,543 & 391 & ,545 & & & ,366 & ,568, & ,390 & 557, \\
\hline 9 & ,517 & & & & & & & & & \\
\hline 10 & ,713 & & 699, & & 711, & & 708, & & 719, & \\
\hline 11 & ,720 & & & & & & & & & \\
\hline 12 & 673, & & ,701 & & 702, & & 700 & & ,715 & \\
\hline 13 & ,417 & ,386 & ,401 & ,403 & & & & & & \\
\hline 14 & ,442 & ,356 & 493, & ,311 & & & & & & \\
\hline 15 & & ,662 & & 659, & & 696, & & 696, & & 699, \\
\hline 16 &, 593 & ,338 & ,622 & ,311 & & & & & & \\
\hline
\end{tabular}

* Resultado da mesma seqüência de análises nos dados não-transformados.

A validação convergente e discriminante foi realizada pela correlação entre o MAC-Q e as escalas prospectiva e retrospectiva da versão em português do PRMQ, chamada PRMQ-10 por possuir 10 itens, sendo cinco de cada escala. As análises foram realizadas com 38 participantes com idade entre 60 e 81 anos, sendo a idade média de 69,03 anos (DP = 5,27).

Os escores do MAC-Q correlacionaram-se positivamente somente com a escala retrospectiva do PRMQ ( $r=0,46, p<0,05)$. Essa correlação é considerada uma evidência de que a escala retrospectiva do PRMQ avalia, aproximadamente, o mesmo traço que o MACQ, ou seja, percepção de falhas de memória retrospectiva. A falta de correlação entre o MAC-Q e a escala prospectiva do PRMQ-10 indica a validade discriminante dessa escala, e a falta de correlação entre o MAC-Q e o escore geral do PRMQ-10 aponta para a unidimensionalidade do MAC-Q e bidimensionalidade do PRMQ.

O PRMQ na versão em português com 10 itens apresentou boa confiabilidade no grupo de idosos segundo os índices de consistência interna: alfa de Cronbach igual a 0,87 no PRMQ total, 0,78 na escala prospectiva, 0,76 na retrospectiva, assim como o MAC$\mathrm{Q}$, alfa igual a 0,86 . O cálculo do escore geral do PRMQ-10 representa a quantificação do relato de falhas de memória em geral e pode ser realizado a partir da soma de todos os itens que compõem a escala, ou seja, dos itens $1,2,3,4,5,6,8,10,12$ e 15 . O escore da escala prospectiva pode ser calculado pela soma dos itens $1,3,5,10$ e 12 e o escore da escala retrospectiva pela soma dos itens 2, 4, 6, 8 e 15. 
Tabela 3 - Freqüência das respostas ao PRMQ

\begin{tabular}{|c|c|c|c|c|c|c|c|c|c|c|c|c|}
\hline \multirow{3}{*}{$\begin{array}{c}\text { Itens } \\
\text { do } \\
\text { PRM } \\
\text { Q }\end{array}$} & \multicolumn{10}{|c|}{ Percentis das respostas $(\%)$} & \multirow{2}{*}{\multicolumn{2}{|c|}{ Médias e desvios padrão }} \\
\hline & \multicolumn{2}{|c|}{ 1. Nunca } & \multicolumn{2}{|c|}{ 2. Raramente } & \multicolumn{2}{|c|}{$\begin{array}{l}\text { 3. Algumas } \\
\text { vezes }\end{array}$} & \multicolumn{2}{|c|}{$\begin{array}{l}\text { 4. Freqüente- } \\
\text { mente }\end{array}$} & \multicolumn{2}{|c|}{$\begin{array}{l}\text { 5. Quase } \\
\text { sempre }\end{array}$} & & \\
\hline & Geral & Idoso & Geral & Idoso & Geral & Idoso & Geral & Idoso & Geral & Idoso & Geral & Idoso \\
\hline 1 & 6,9 & 7,9 & 33,9 & 18,4 & 50,5 & 55,3 & 6,0 & 2,6 & 2,7 & 15,8 & $2,64 \pm 0,80$ & $3,00 \pm 1,09$ \\
\hline 2 & 43,6 & 37,8 & 44,8 & 32,4 & 9,0 & 18,9 & 1,8 & 5,4 & 0,8 & 5,4 & $1,71 \pm 0,76$ & $2,08 \pm 1,14$ \\
\hline 3 & 24,5 & 10,8 & 41,4 & 27,0 & 26,3 & 37,8 & 6,5 & 18,9 & 1,4 & 5,4 & $2,19 \pm 0,92$ & $2,81 \pm 1,05$ \\
\hline 4 & 25,3 & 27,0 & 39,6 & 27,0 & 26,7 & 27,0 & 7,0 & 16,2 & 1,3 & 2,7 & $2,19 \pm 0,94$ & $2,41 \pm 1,14$ \\
\hline 5 & 24,5 & 25,0 & 40,5 & 33,3 & 26,4 & 30,6 & 6,3 & 2,8 & 2,3 & 8,3 & $2,21 \pm 0,95$ & $2,36 \pm 1,15$ \\
\hline 6 & 45,3 & 27,8 & 36,3 & 33,3 & 13,6 & 19,4 & 3,7 & 8,3 & 1,0 & 11,1 & $1,79 \pm 0,88$ & $2,42 \pm 1,29$ \\
\hline 7 & 37,9 & 30,6 & 35,9 & 33,3 & 21,2 & 22,2 & 3,8 & 2,8 & 1,2 & 11,1 & $1,95 \pm 0,92$ & $2,31 \pm 1,26$ \\
\hline 8 & 29,5 & 41,7 & 38,2 & 11,1 & 24,8 & 36,1 & 6,6 & 5,6 & 0,9 & 5,6 & $2,11 \pm 0,93$ & $2,22 \pm 1,22$ \\
\hline 9 & 30,4 & 30,6 & 37,9 & 25,0 & 25,0 & 30,6 & 5,5 & 11,1 & 1,1 & 2,8 & $2,09 \pm 0,93$ & $2,31 \pm 1,11$ \\
\hline 10 & 16,9 & 13,9 & 36,9 & 30,6 & 36,5 & 36,1 & 8,1 & 13,9 & 1,5 & 5,6 & $2,40 \pm 0,91$ & $2,67 \pm 1,06$ \\
\hline 11 & 13,9 & 13,9 & 36,1 & 11,1 & 35,5 & 36,1 & 10,7 & 19,4 & 3,8 & 19,4 & $2,54 \pm 0,98$ & $3,19 \pm 1,28$ \\
\hline 12 & 20,1 & 29,7 & 44,3 & 29,7 & 28,2 & 21,6 & 5,2 & 8,1 & 2,3 & 10,8 & $2,25 \pm 0,91$ & $2,41 \pm 1,30$ \\
\hline 13 & 36,0 & 55,6 & 39,9 & 16,7 & 20,1 & 16,7 & 3,5 & 8,3 & 0,5 & 2,8 & $1,92 \pm 0,86$ & $1,86 \pm 1,15$ \\
\hline 14 & 33,0 & 16,2 & 41,9 & 45,9 & 20,9 & 27,0 & 3,1 & 5,4 & 1,2 & 5,4 & $1,98 \pm 0,87$ & $2,38 \pm 1,01$ \\
\hline 15 & 32,5 & 40,5 & 39,8 & 16,2 & 21,8 & 27,0 & 4,6 & 10,8 & 1,2 & 5,4 & $2,02 \pm 0,91$ & $2,24 \pm 1,25$ \\
\hline 16 & 10,5 & 16,7 & 39,7 & 30,6 & 40,2 & 36,1 & 8,5 & 8,3 & 1,1 & 8,3 & $2,50 \pm 0,83$ & $2,61 \pm 1,12$ \\
\hline Médias & 26,87 & 26,60 & 39,19 & 26,35 & 26,67 & 29,90 & 5,68 & 9,24 & 1,54 & 7,87 & $2,15 \pm 0,89$ & $2,45 \pm 1,16$ \\
\hline
\end{tabular}

Tabela 4 - Teste de validade convergente e discriminante

\begin{tabular}{llll}
\hline & 1 & 2 & 3 \\
\hline 1. Relato de falhas de memória (PRMQ-10) & - & & \\
2. Escala Prospectiva &, $912^{* *}$ & - & - \\
3. Escala Retrospectiva &, $935^{* *}$ &, $731^{* *}$ &, $461^{*}$ \\
4. MAC-Q do Idoso &, 360 &, 329 & \\
\hline
\end{tabular}

** Correlação é significativa no nível $p<0,01$ (2-tailed).

* Correlação é significativa no nível $p<0,05$ (2-tailed).

\section{Discussão}

O processo de tradução e adaptação cultural abrangeu etapas importantes para a análise da pertinência e utilização do PRMQ na versão em português. Essas fases de análise do instrumento são evidenciadas como necessárias para o uso de instrumentos originados em outras culturas (Pasquali, 2001). Já que os resultados da análise de construto revelaram que vários itens não discriminaram as dimensões estruturais do PRMQ (prospectiva e retrospectiva), frisa-se a necessidade de as fases de tradução e adaptação serem novamente avaliadas antes de posteriores pesquisas sobre a validade do instrumento.

As propriedades do PRMQ versão em português permaneceram semelhantes às do instrumento original, pois a metade dos itens (oito) formou dois fatores distintos, de acordo com as dimensões apresentadas por Smith e cols. (2000). A distribuição dos itens em dois fatores apontou para a distinção entre os processos subjacentes à memória prospectiva e retrospectiva, e a possibilidade dessa diferença ser representada semanticamente (Brandimonte e cols., 2001; Parente e cols., 2005).

Porém, os itens que migraram de fator, 9 e 11, descrevem situações de uso de memória teoricamente congruentes com o fator que representam, conforme foi verificado pela validade de face realizada previamente à coleta de dados. $O$ item 9 não foi alterado na tradução para o português e os participantes não apresentaram dificuldade de interpretação. $\mathrm{O}$ item 11 foi adaptado, fato que pode ser a causa da migração de dimensão. Nesse item, a substituição da palavra "confunde" pela palavra "esquece" reforça o vínculo teórico da variável ao 
conceito de memória retrospectiva e assegura a validade de face do instrumento. Esse padrão pode ter influenciado a formação dos fatores no modelo do PRMQ na versão em português, pois falhas de memória prospectiva ocorrem mais comumente do que falhas de memória retrospectiva (McDaniel \& Einstein, 2000). A situação descrita no item 11 pode ter sido interpretada como mais propensa à ocorrência de falhas do que seria com o verbo confunde.

Uma hipótese geral para a migração dos dois itens aponta para o auto-relato de falhas mais pronunciadas nesses itens em relação aos outros itens retrospectivos. Ou seja, os itens retrospectivos que descrevem situações nas quais ocorrem muitas falhas (9 e 11) aproximaram-se dos itens prospectivos, nos quais são esperadas, teoricamente, freqüências de falhas mais altas. Outras hipóteses para a migração dos itens apontam para a má construção das frases no instrumento original, má tradução e adaptação na língua portuguesa ou para a impossibilidade de generalização do construto em diferentes línguas mediante essas variáveis. Neste último caso seria necessário construir novos itens para um instrumento em português com os fundamentos teóricos que embasam o PRMQ.

Os primeiros resultados da análise fatorial dos 16 itens do PRMQ revelaram que os itens 9 e 11 não devem ser utilizados, pois não apresentaram validade de construto na versão em português. A realização de novas análises causou uma outra alocação das variáveis nas dimensões prospectiva e retrospectiva. $\mathrm{O}$ item três, que estava dividido, ficou alocado no fator correspondente ao modelo original (prospectivo) e o item quatro, que era característico do fator retrospectivo, apresentou uma tendência de divisão entre os fatores. Novas análises foram realizadas após a exclusão dos itens divididos, já que estes diminuem a confiabilidade na validade de construto da escala.

A análise realizada com os oito itens que apresentaram boa validade de construto confirmou a eficácia da escala para quantificar auto-relato de falhas de memória com base em duas dimensões de memória, prospectiva e retrospectiva. Em virtude da necessidade de equivalência entre o número de variáveis prospectivas e retrospectivas para a utilização prévia do PRMQ, foram incluídos dois itens divididos à escala retrospectiva. Esse recurso é bastante comum na adaptação de instrumentos, por afastar a possibilidade de a adaptação gerar um instrumento estruturalmente diferente do original (Blaya, 2005). Novas análises com a presença dos dois itens divididos apontaram para a contribuição desses nas duas dimensões, uma vez que a performance das outras variáveis não foi afetada significativamente.
A partir desses resultados, aconselha-se o uso do PRMQ com 10 itens (PRMQ-10), sendo cinco itens prospectivos e cinco retrospectivos, até que novas pesquisas investiguem os itens que não apresentaram boa validade de construto na versão em português. Salienta-se que o PRMQ-10 possui dois itens retrospectivos que possuem somente validade de face, e uma tendência para o fator retrospectivo. A interpretação dos escores produzidos pelo PRMQ-10 versão em português deve ser realizada com cautela, principalmente quanto à dimensão retrospectiva.

A redução do número de itens do PRMQ impossibilitou a avaliação do relato de falhas de memória quanto às subdimensões do questionário: de tempo (curto e longo prazo) e pista (externa e interna) (Einstein e cols., 1995; Smith e cols., 2000). O diferente número de itens em cada escala, provocado pela exclusão de itens do PRMQ, impediu a avaliação dessa faceta do instrumento. A utilização de dados provenientes das subdimensões representa uma potencialidade do PRMQ que pode ser utilizada a partir da continuidade de aperfeiçoamento e adaptação do questionário.

Os testes de validade convergente e discriminante refletiram a tendência à unidimensionalidade do MAC-Q (Crook e cols., 1992) formado por cinco itens retrospectivos e somente um, aparentemente, prospectivo (item 5). A correlação específica entre a escala retrospectiva do PRMQ-10 e o MAC-Q apontou para a validade convergente da escala retrospectiva e para a validade discriminante da escala prospectiva do PRMQ-10 na versão em português. Salienta-se que esses resultados foram encontrados entre o grupo de idosos (60 a 81 anos).

A partir da apresentação dos índices prévios de validade e confiabilidade do PRMQ-10 e suas escalas, o instrumento pode ser utilizado na sua versão em português com dez itens, desde que ressaltado a fato de o processo de adaptação não estar completamente concluído. O PRMQ-10 pode ser utilizado na clínica, em triagens, hospitais e em pesquisas sobre auto-relato de falhas de memória prospectiva e retrospectiva em populações de língua portuguesa. Em especial, o PRMQ-10 avalia o relato de falhas de memória prospectiva, não contemplada por nenhum instrumento disponível em português anteriormente.

Pesquisas de adaptação de instrumentos devem ser cautelosas e permanecer fidedignas quanto às características originais do instrumento, pois interessa a adaptação e não a construção de um instrumento novo (Pasquali, 2001). Outras investigações devem ser realizadas a fim de que as características psicométricas do PRMQ sejam avaliadas em populações clínicas e com cuidadores de idosos, quando o instrumento seria 
respondido na terceira pessoa, e informaria as falhas de memória de acordo com a percepção de quem cuida. Outros tipos de validade e confiabilidade também podem ser testados, como a validade empírica, validade clínica, e a confiabilidade teste-reteste.

\section{Referências}

Anastasi, A. \& Urbina, S. (2000). Testagem psicológica. (M. A. V. Veronese, Trad.). Porto Alegre: Artes Médicas Sul.

Argimon, I. L. \& Stein, L. M. (2005). Habilidades cognitivas em indivíduos muito idosos: um estudo longitudinal. Caderno de Saúde Pública do Rio de Janeiro, 21(1), 64-72.

Blaya, C. (2005). Tradução, adaptação e validação do Defensive Style Questionnaire (DSQ-40) para o português brasileiro. (Dissertação de Mestrado). Porto Alegre: Universidade Federal do Rio Grande do Sul - Curso de Pós-Graduação em Psiquiatria.

Brandimonte, M. A., Ferrante, D., Feresin, C. \& Delbello, R. (2001). Dissociating prospective memory from vigilance process. Psicológica, 22, 97113.

Callegari-Jacques, S. M. (2004). Bioestatística: princípios e aplicações. Porto Alegre: Artmed.

Cherry, K. E., Martin, R. C., Simmons-D’Gerolamo, S. S., Pinkston, J. B., Griffing, A. \& Gouvier, W. D. (2001). Prospective remembering in younger and older adults: Role of the prospective cue. Memory, 9, 177-193.

Crawford, J. R., Smith, G., Maylor, E. A., Sala, S. D. \& Logie, R. H. (2003). The Prospective and Retrospective Memory Questionnaire (PRMQ): Normative data and latent structure in a large nonclinical sample. Memory, 11(3), 261-275.

Crook, T. H., Feher, E. P. \& Larrabee, G. J. (1992). Assessment of memory complaints in age-associated memory impairment: The MAC-Q. International Psychogeriatry, 4, 165-176.

Einstein, G. O., McDaniel, M. A., Richardson, S. L., Guynn, M. J. \& Cunfer, A. R. (1995). Aging and prospective memory: Examining the influences of self-initiated retrieval processes. Journal of Experimental Psychology: Learning, Memory and Cognition, 21, 9961007.

Fachel, J. M. G. \& Camey, S. (2000). Avaliação psicométrica: a qualidade das medidas e o entendimento dos dados. Em J. A. Cunha (Org.). Psicodiagnóstico $V$ (pp. 158-170). Porto Alegre: Artmed.

Henry, J. D., MacLeod, M. S., Phillips, H. \& Crawford, J. R. (2004). A meta-analytic review of prospective memory and aging. Psychology and Aging, 19(1), 27-39.

Marsh, R., L., Hicks, J. L. \& Watson, V. (2002). The dynamics of intention retrieval and coordination of action in event-based in prospective memory. Journal of Experimental Psychology: Learning, Memory, and Cognition, 28(4), 652-659.

McDaniel, M. A. \& Einstein, G. O. (2000). Strategic and automatic processes in prospective memory retrieval: A multiprocess framework. Applied Cognitive Psychology, 14, 127-144.

Parente, M. A. M. P., Taussik, I. M., Ferreria, E. D. \& Kristensen, C. H. (2005). Different patterns of prospective, retrospective, and working memory decline across adulthood. Revista Interamericana de Psicologia, 39(2), 231-238.

Pasquali, L. (2001). Técnicas de exame psicológico: TEP: manual. São Paulo: Casa do Psicólogo.

Smith, G., Sala, D. S., Logie, R. \& Maylor, E. A. (2000). Prospective and retrospective memory in normal aging and dementia: A questionnaire study. Memory, 8(5), 311-321.

Sunderland, A., Harris, J. E., \& Baddeley, A. D. (1988). Do laboratory tests predict everyday memory? A neuropsychological study. Journal of Verbal Learning and Verbal Behavior, 22, 341-357.

Tabachnich, B. G. \& Fidell, L. S. (2001). Using Multivariate Statistics. Needham Heights, MA: Allyn and Bacon.

Recebido em junbo de 2006

Reformulado em março de 2007

Aprovado em abril de 2007 
Sobre os autores:

Daniela Benites concluiu o Curso de Psicologia na Universidade Regional Integrada - Câmpus de Santo Angelo RS, ingressando a seguir no Programa de Pós-Graduação em Psicologia da Universidade Federal do Rio Grande do Sul, onde concluiu o Mestrado e cursa atualmente o doutorado sob orientação de W. B. Gomes. O presente trabalho faz parte de sua dissertação de Mestrado.

William B. Gomes faz parte do grupo fundador do Programa de Pós-Graduação em Psicologia da Universidade Federal do Rio Grande do Sul, onde leciona disciplinas relacionadas à Epistemologia e História da Psicologia, e coordena o Laboratório de Cognição e Fenomenologia Experimental. Foi também fundador e primeiro editor da Revista Psicologia: Reflexão e Crítica. É bolsista de produtividade 1A do CNPq. 